\title{
DESEMPENHO FUNCIONAL MELHOR INDICADOR DE MORTALIDADE DO QUE AUTOPERCEPÇÃO DE SAÚDE EM NONAGENÁRIOS E CENTENÁRIOS
}

Vivian Ulrich; Grupo de Atenção Multiprofissional Domiciliar ao Longevo (AMPAL) da PUCRS, Porto

Alegre, Brasil; viviulrich@gmail.com

Victória Albino Araujo, Escola de Medicina da PUCRS, Porto Alegre, Brasil; victoria.albino.araujo@gmail.com

Aline Mendes da Rosa, Grupo de Atenção Multiprofissional Domiciliar ao Longevo (AMPAL) da PUCRS, Porto Alegre, Brasil; alinehaco@gmail.com

Ângelo José Gonçalves Bós, Escola de Medicina da PUCRS, Porto Alegre, Brasil; angelo.bos@pucrs.br

\section{Resumo}

Introdução: Pouco é estudado sobre a mortalidade e qualidade de vida de nonagenários e centenários. A qualidade de vida pode ser medida pela autopercepção de saúde ou pela capacidade de realizar atividades de vida diária (funcionalidade). Objetivos: O presente estudo se propõe a avaliar a importância da funcionalidade e da autopercepção de saúde como indicadores de sobrevida em nonagenários e centenários. Métodos: Participaram 223 nonagenários e centenários avaliados em 2016 e acompanhados até setembro de 2019. A funcionalidade subjetiva foi medida pela facilidade ou incapacidade em realizar 12 atividades (funcionais e básicas) de vida diária. O desempenho funcional objetivo foi medido pelo teste timed-up-and-go (TUG). Resultados: A percentagem de participantes com autopercepção de saúde "mal/péssima" foi maior entre os falecidos, embora não significativamente associada à sobrevida $(\mathrm{p}=0,1432)$. Foram significativamente associados à sobrevida o melhor desempenho nas atividades funcionais $(\mathrm{p}<0,001)$, básicas $(\mathrm{p}<0,001)$ e TUG $(\mathrm{p}<0,001)$. Somente as atividades básicas perderam a significância na análise ajustada, indicando que o desempenho em atividades funcionais é mais importante na predição de sobrevida. $\mathrm{Na}$ análise ajustada ter menos de 95 anos de idade também deixou de ser significativo e sexo passou a ter nível indicativo de significância estatística $(\mathrm{p}=0,0660)$. Conclusões: Portanto, nonagenários e centenários terão sobrevida semelhante se tiverem o mesmo nível de funcionalidade, tanto subjetiva quanto objetiva, e a autopercepção de saúde não foi um indicador significativo. Homens, nonagenários e centenários, são mais vulneráveis a perdas funcionais do que as mulheres.

Palavras-chave: Longevidade; Envelhecimento; Funcionalidade; Mortalidade; Sobrevida.

Agradecimento: O presente trabalho foi realizado com apoio da Coordenação de Aperfeiçoamento de Pessoal Nível Superior - Brasil (CAPES) - Código de Financiamento 001. 